\title{
Pharmaciana
}

Vol.11, No.2, July 2021, Page. 283-292

ISSN: 2088 4559; e-ISSN: 24770256

DOI: $10.12928 /$ pharmaciana.v11i2.20674

\section{Antioxidant-phenolic content correlation of phenolics rich fractions from Dillenia suffruticosa wood bark}

\author{
Rini Muharini*, Ira Lestari, Masriani \\ Department of Chemistry Education, Faculty of Teacher Training and Education, Universitas Tanjungpura \\ Jl. Prof. Dr. H. Hadari Nawawi, Pontinak, West Kalimantan, Indonesia
}

Submitted: 11-05-2021

Reviewed: 30-05-2021

Accepted: 15-06-2021

\begin{abstract}
Dillenia suffruticosa is one of the tropical medicinal plants that has been used traditionally for treating several tropical diseases. However, phytochemical and pharmacological investigations of $D$. suffruticosa wood bark have not been well-investigated. This research aimed to investigate the correlation between antioxidant properties and total phenolic content of phenolics rich extract and fractions from $D$. suffruticosa wood bark. The phytochemical investigation revealed the existence of phenolics and alkaloids in methanolic crude wood bark extract and all fractions. Meanwhile, the presence of flavonoids was shown in chloroform and methanol fractions. The highest total phenolic content was demonstrated by methanol fraction with $254.34 \pm 16.86 \mathrm{mg}$ GAE/g extract. Meanwhile, the highest total flavonoid content was interestingly displayed in chloroform fraction with $15.33 \pm 0.26$ $\mathrm{mg} \mathrm{RE} / \mathrm{g}$ extract. The crude extract and methanol fraction had a profound antioxidant activity with $\mathrm{IC}_{50}$ values of less than $15.63 \mathrm{ppm}$ and $8.83 \mathrm{ppm}$, respectively. A strong correlation was shown between the antioxidant activity and total phenolic contents through correlation analysis. Thus, the $D$. suffruticosa wood bark could be considered a potential natural source of antioxidants.
\end{abstract}

Keywords: Dillenia suffruticosa wood bark, antioxidant activity, total phenolics, total flavonoids, antioxidant and phenolic content correlation

\footnotetext{
*Corresponding author:

Rini Muharini

Department of Chemistry Education, Faculty of Teacher Training and Education, Universitas Tanjungpura

J1. Prof. Dr. H. Hadari Nawawi, Pontinak, West Kalimantan, Indonesia

Email: rini.muharini@fkip.untan.ac.id
} 


\section{INTRODUCTION}

West Kalimantan is known as the habitat for tropical medicinal plants with swamp forest ecology. The uniqueness of the ecology would influence the ability of plants or organisms to produce secondary metabolites (Kessler and Kalske, 2018). Local people mostly utilize this biodiversity for their daily routine activities, such as for traditional medicine. One of these plants is Dillenia suffruticosa, known as Simpoh by Dayak tribe. The D. suffruticosa, which belongs to the Dilleniaceae family, is a native plant to the Malay peninsula, Indonesia, and the Philippines. Traditionally, this species is used for therapeutic purposes. Alongside its usage as wrappers for Tempe, a fermented beans food product, the leaf of $D$. suffruticosa was believed to treat inflammation (Ahmad and Holdsworth, 1995), bleeding cough (Gunadi et al., 2017), rheumatism, and fever (Yazan and Armania, 2014). Meanwhile, the fruits were applied for treating painful areas caused by suspected cancer (Ahmad and Holdsworth, 1995). Pharmacological investigation to some parts of this plant showed that the leaves extract showed antimicrobial (Wiart et al., 2004), antioxidant, antivirus Dengue, and antifungal activities (Yazan and Armania, 2014). Meanwhile, the fruit extract displayed antioxidant and cytotoxic activities toward Hela and HT29 cancer cell lines (Armania et al., 2013). In addition, methanolic extract from the roots exhibited antioxidant activity, cytotoxic activities against colon cancer HT29, breast cancer MCF-7 and MDA-231, ovarian cancer CaOV3, lung cancer A549, and cervix cancer Hela cells (Armania et al., 2013; Tor et al., 2014).

Many studies have revealed that antioxidant agents were found to display other medicinal properties, such as anti-inflammatory (Chen et al., 2019), cytotoxicity (Gacche and Jadhav, 2012), or anticancer (Grigalius and Petrikaite, 2017). It suggested that the antioxidant mechanism can inhibit cellular damage by free radicals (Chen et al., 2019). The fact that all investigated part plants were reported to possess antioxidant property gives the hypothesis that the wood bark would have the same antioxidant property. Thus, it would have other interesting properties. Yet, hitherto no reports exist on the biological activity of the wood bark of $D$. suffruticosa.

Previously, a phytochemical screening on leaves, roots, fruits, and flowers of D.suffruticosa reported the presence of saponins, triterpenes, sterols, and polyphenols (Armania et al., 2013). Tor group (2015) worked on D. suffruticosa roots and isolated: two flavonoids (kaempferide and kaempferol), two phenolics (protocatechuic acid and gallic acid), and two triterpenoids (3-Epimaslinic acid and $\beta$-sitosterol-3-O- $\beta$-D-glucopyranoside). Further investigation on $D$. suffruticosa leaves reported isolation flavonoids of vitexin, tiliroside, and kaempferol, as well as triterpenoids of betulinic acid and koetjapic acid (Abubakar et al., 2019). Nevertheless, there is no literature reporting phytoconstituents from $D$. suffruticosa wood bark to date.

As mentioned above, the extract of leaves, fruits, roots, and flowers have been investigated and shown a very promising plant for a biologically active natural product source. Yet, there are hitherto no studies concerned with phytoconstituents from D. suffruticosa wood bark and their biological evaluation. Since $D$. suffruticosa plants are abundant in West Kalimantan and found to be important pharmacologically, it is essential to investigate the wood bark of D. suffruticosa in order to reveal their phytochemical and antioxidant properties. This paper will describe qualitative phytochemical investigation on the extract $D$. suffruticosa wood bark, determination of total phenolic contents (TPC) and total flavonoid content (TFC), the antioxidant property of the extract and its fractions, and total phenolic content-antioxidant correlation.

\section{MATERIALS AND METHOD Materials}

A dual-beam Ultra Violet/Visible (UV/Vis) spectrophotometer (Rayleigh UV-2601, China) was utilized to measure the absorbances of anti-oxidative fractions using DPPH method, the complex Phenolic-Folin, and the complex aluminum-flavonoid. Silica gel 60 (230-400 mesh) for column chromatography (Merck Millipore, USA) and Silica gel 60 (30-70 mesh) for column chromatography 
(Merck Millipore, USA) were used for Vacuum Liquid Chromatography (VLC) and Gravitational Column Chromatography (GCC), respectively. Silica gel 60 F254 on Thin Layer Chromatography (TLC) aluminum plate (Merck Millipore, USA) was employed to observe the extraction process, to recognize the type of secondary metabolites, and to conduct qualitative antioxidant activity using TLC technique. The chromatograms on TLC plate were detected using spray reagent $\mathrm{CeSO}_{4} 1.5 \%$ for phytochemical test and using DPPH spray reagent for the qualitative antioxidant test. All fractions were evaporated using a rotary-vacuum evaporator (Buchi, Germany). Distilled and pro-analysis solvents were employed for extraction, identification, and spectroscopic measurement.

The wood barks of D.suffruticosa collected in May 2017 at Pontianak Timur district, Pontianak, were used as plant material. The plant voucher was determined and deposited at Research Center for Biology, Cibinong, Indonesia, with letter number 2003/IPH.

\section{Extraction and isolation}

The wood barks of D.suffruticosa were air-dried and granulated. The powdered D. suffruticosa wood bark $(2.5 \mathrm{~kg})$ was extracted with methanol $(3 \times 24 \mathrm{~h})$ to give a methanolic crude extract. The crude extract was partitioned with $n$-hexane and chloroform to give $n$-hexane, chloroform and methanol fractions. Each fraction was evaporated to yield $n$-hexane $(3.2 \mathrm{~g})$, chloroform $(7.02 \mathrm{~g})$, and methanol $(73.8 \mathrm{~g})$ fractions.

\section{Phytochemical test}

The phytochemical test was performed on all fractions using natural product reagents, including $\mathrm{FeCl}_{3} 5 \%$, Shinoda, Mayer, Dragendorf, Liebermann-Burschad, and Salwkosky. Spots on TLC were detected by applying spray reagent $\mathrm{CeSO}_{4} 1,5 \%$ and subsequently heating the TLC plate on the hot plate (Sticher, 2008).

\section{Antioxidant test}

The antioxidant activity test was conducted using the 2,2-diphenyl-1-picrylhydrazyl (DPPH) method toward methanolic crude extract, $n$-hexane, chloroform, and methanol fractions as tested samples. The positive control used was Vitamin C. The DPPH method conducted was altered from the previous antioxidant protocol by using TLC-bioautograph technique (Cies̈la et al., 2015) and UV-Vis spectrophotometry (Salazar-aranda et al., 2011).

\section{Qualitative antioxidant test}

A qualitative antioxidant test was conducted using TLC-bioautography technique described previously by Cië̈la (Cies̈la et al., 2015) with minor modification. $50 \mathrm{ml}$ DPPH solution $(20 \mu \mathrm{g} / \mathrm{mL})$ was prepared as DPPH spray reagent and then stored in dark room. $10 \mu$ of each sample $(1 \mathrm{mg} / \mathrm{ml}$ in methanol) were applied on TLC plate together with positive controls, Vitamin C and E, which were placed side by side with samples. After spraying the TLC plate containing spots from sampled and positive control with DPPH solution, it was directly set aside in a dark room for 30 minutes. The white or yellow spot on the purple TLC plate was spotted to indicate antioxidant property. The same protocol was applied to each sample and positive control with concentrations ranging from 125 to $1000 \mathrm{ppm}$.

\section{Quantitative antioxidant test}

A quantitative antioxidant test was carried out using the UV-Vis spectrophotometry technique protocol described by Salazar-aranda (Salazar-aranda et al., 2011) with slight modification. $10 \mathrm{~mL}$ stock solution in $1 \mathrm{mg} / \mathrm{mL}$ of each sample was made ready in methanol. $50 \mathrm{ml}$ of vitamin $\mathrm{C}(0.5$ $\mathrm{mg} / \mathrm{mL}$ ) as a positive control was prepared. $100 \mu \mathrm{l}$ of each sample in a concentration of $1000-1.95$ ppm (except for crude extract, concentration ranging from $1000-15.625 \mathrm{ppm}$ ) was added with $100 \mu \mathrm{l}$ DPPH solution in methanol $(0.004 \%)$. The mixture was blended and kept aside in a dark room at 
room temperature for 30 minutes. Observation on the absorbance of each fraction at wavelength 517 $\mathrm{nm}$ was done using a UV-Vis spectrophotometer. The absorbance value was then changed into a percentage of antioxidant activity (AA\%) using formula as shown in equation (1),

$$
\mathrm{AA} \%=((A-B) \div A) \times 100
$$

(1)

where A is the absorbance of control and B is the absorbance of the sample. The negative control is the mixture of DPPH and methanol, while the sample is DPPH, methanol, and sample.

A blank solution (Abs blank) used was $200 \mu \mathrm{l}$ methanol. The control solution (Abs control) was a mixture of $100 \mu \mathrm{LPPH}(0.004 \%)$ and $100 \mu \mathrm{l}$ methanol. The antioxidant activity was conveyed in a percent concentration required to reduce $50 \% \mathrm{DPPH}$ absorbance $\left(\mathrm{IC}_{50}\right)$. The $\mathrm{IC}_{50}$ value was calculated with a linear regression equation between the percentages of antioxidant activity as a function of sample concentration. Each measurement was done in triplicate, and the same protocol was done with the vitamin $\mathrm{C}$ (positive control).

\section{Determination of total phenolics content (TPC)}

An experiment using the Folin-Ciocalteu method described by Armania (2013) with some modification was conducted to determine total phenolics content on each extract and fractions. In brief, $0.5 \mathrm{ml}$ tested sample $(1 \mathrm{mg} / \mathrm{mL})$ was reacted with $2.5 \mathrm{~mL}$ Folin-Ciocalteu and then was added with $2 \mathrm{~mL}$ of $\mathrm{Na}_{2} \mathrm{CO}_{3} 7.5 \%$. The mixture was incubated at $40^{\circ} \mathrm{C}$ for an hour. The absorbance of the mixture was then measured using UV Spectrophotometry at $\lambda 765 \mathrm{~nm}$. The experiment was done in triplicate. The positive control was gallic acid, with a standard linear curve of gallic acid is $\mathrm{Y}=0.0129$ $\mathrm{X}-0.0243\left(\mathrm{R}^{2}=0.9977\right)$. The total phenolics content was demonstrated as milligram gallic acid equivalent per gram extract (mgGAE/g extract)

\section{Determination of total flavonoids content (TFC)}

Determination of total flavonoid content was performed by using the aluminum chloride method described by Armania (2013) with some modifications. A $10 \mu \mathrm{l}$ of tested sample $(1 \mathrm{mg} / \mathrm{mL})$ was added with $100 \mu \mathrm{l}$ of $\mathrm{AlCl}_{3} 2 \%$, and let the mixture at room temperature for 10 minutes. The absorbance of complex flavonoid-aluminum was observed using UV spectrophotometry at $\lambda 405 \mathrm{~nm}$ and was done in triplicate. Rutin was used as a positive control. The TFC was displayed as a milligram of rutin equivalent per gram extract (mg RE/g extract).

\section{Statistical analysis}

All data were measured in triplicate. TPC and TFC values were represented as mean \pm standard deviation. The mean difference between crude extract, $n$-hexane, chloroform, and methanol fractions was tested using a one-way analysis of variance with $\mathrm{p}<0.05$. Pearson's product-moment correlation was performed to determine the correlation coefficient.

\section{RESULT AND DISCUSSION}

The powdered D. suffruticosa wood bark $(2.5 \mathrm{~kg})$ was macerated with methanol solvent three times for $24 \mathrm{~h}$; each was given to $84.1 \mathrm{~g}$ of crude extract (M). Repeated partition using liquid-liquid extraction toward crude extract with $n$-hexane and chloroform, followed by rotary-vacuum evaporation, yielded $n$-hexane (KSH) concentrate $(3.2 \mathrm{~g}$ ), chloroform (KSC) (7.02 g), and methanol (KSM) $(73.8 \mathrm{~g}$ ) fractions (Figure 1). These yields showed that the most abundant secondary metabolite from the wood bark extract of D.suffruticosa was found in methanol fraction, indicating that most of them are polar compounds.

Phytochemical screening on methanolic crude extract and all fractions were done using specific reagents. Those specific reagents were for alkaloids, flavonoids, phenolics, triterpenoids, saponin, and 
sterols screening. Phytochemical test on the crude extract results alkaloids, phenolics, flavonoids, triterpenoids, and sterols (Table 1). Although the methanolic crude extract gave negative results for saponin, the chloroform and methanol fraction gave positive results for this group. Methanol fraction, which has the highest amount, gave positive results for phenolics, alkaloids, flavonoids, and saponins. The presence of alkaloids and phenolics was also shown in n-hexane and chloroform fractions, indicating that these secondary metabolites dominated the extract of $D$. suffruticosa wood bark. The presence of phenolics in all fractions corresponds with previous phytochemical studies on other parts of $D$. suffruticosa. Recently, an intensive investigation was conducted toward D. suffruticosa leaves, and it reported the existence of alkaloids from this part of the plant (Dawood Shah et al., 2020). Like an alkaloid that was detected in leaves, an alkaloid from D. suffruticosa wood bark was strongly detected. This finding is interesting because it indicated that alkaloids were also specifically deposited in the wood bark. However, there were no reports regarding alkaloids that isolated from $D$. suffruticosa so far. On the other side, flavonoid was likely not a major component in $D$. suffruticosa wood bark. Based on this data, it seems that the diversity of secondary metabolites on the wood bark of D.suffruticosa was high.

Table 1. Phytochemical test results for crude extract, $n$-hexane, chloroform, and methanol fractions of D. suffruticosa

\begin{tabular}{lllll}
\hline Groups & M & KSH & KSC & KSM \\
\hline Alkaloid & ++ & ++ & ++ & ++ \\
Phenolics & ++ & + & ++ & ++ \\
Flavonoids & + & - & + & + \\
Triterpenoids & + & + & + & - \\
Sterols & + & + & + & - \\
Saponin & - & - & + & + \\
\hline
\end{tabular}

Note: $\mathrm{M}=$ crude extract, $\mathrm{KSH}=n$-hexane fraction, $\mathrm{KSC}=$ chloroform fraction, $\mathrm{KSM}=$ methanol fraction; ++ strongly detected, + detected, - not detected

Antioxidant activity screening using the TLC-bioautography technique with DPPH revealed that methanolic crude extract and methanol fraction gave the brightest spot, contrasting with the purple background. Their spot's color and shape were closely the same with vitamin $\mathrm{C}$ and $\mathrm{E}$ as positive controls (Figure 1). It indicated that methanol fraction was qualitatively potential as an antioxidant (Cies̈la et al., 2015). According to its spot appearance, chloroform fraction was considered to possess slightly active as antioxidant. In this study, antioxidant behaviour of different sample concentrations $(125-1000 \mathrm{ppm})$ was observed using the TLC-bioautography technique and compared to positive control with the same ranging concentrations. The chromatogram showed that the gradation spot patterns of crude extract and methanol fraction are similar to that of vitamin $\mathrm{C}$; even crude extract displayed a brighter spot than vitamin $\mathrm{C}$ at the $125 \mathrm{ppm}$ concentrations (Figure 2). The accumulation of antioxidant agents contained in crude extract might be the reason for these phenomena. Based on the spot appearance of each sample at concentration $125 \mathrm{ppm}$, it seemed that methanol and chloroform fraction would have $\mathrm{IC}_{50}$ values lower than $125 \mathrm{ppm}$. Therefore, the lowest concentration used in a quantitative antioxidant test experiment must be below $125 \mathrm{ppm}$. 


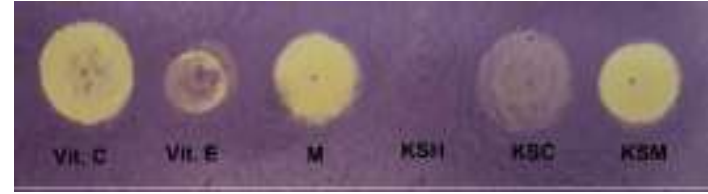

Figure 1. Chromatogram of qualitative antioxidant test for methanol extract $(M), n$-hexane fraction (KSH), chloroform fraction (KSC), methanol fraction (KSM), and vitamin $\mathrm{C}$ and $E$ (positive controls) with concentration $1 \mathrm{mg} / \mathrm{ml}$ each using TLC-based technique and DPPH

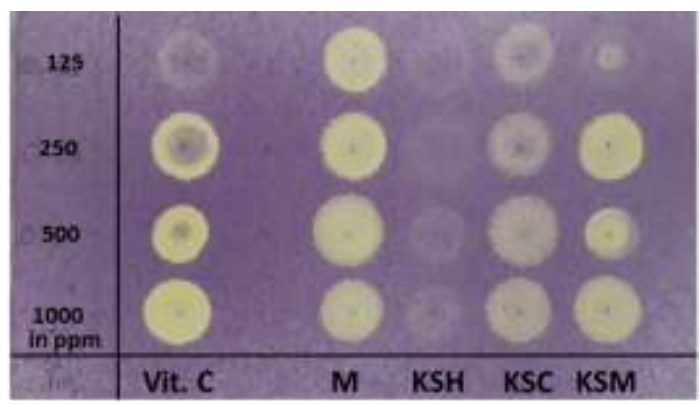

Figure 2. Chromatogram of qualitative antioxidant test using TLC-based technique and DPPH assay for (a) methanol extract (M), $n$-hexane fraction (KSH), chloroform fraction (KSC), methanol fraction (KSM), and vitamin $\mathrm{C}$ (positive controls) with ranging concentration (125 - $1000 \mathrm{ppm})$; ; and (b) methanol fraction eluted with $n$-hexaneacetone (1:1)

The crude extract and all fractions were evaluated further to obtain their antioxidant $\mathrm{IC}_{50}$ values using the UV-Vis spectrophotometry technique. The concentration range was $1000-15.63 \mathrm{ppm}$ for crude extract and $1000-1.95 \mathrm{ppm}$ for the fraction. The crude extract $(\mathrm{M})$ still showed radical inhibition $(82.5 \%)$ at a concentration of $15.63 \mathrm{ppm}$, indicating that the $\mathrm{IC}_{50}$ of the crude extract was less than $15.63 \mathrm{ppm}$. This result demonstrated that the crude extract was very active as an antioxidant, compared to vitamin $\mathrm{C}\left(\mathrm{IC}_{50}=4.45 \mathrm{ppm}\right)$ as the positive control. Chloroform (KSC) and methanol $(\mathrm{KSM})$ fractions exhibited moderate and pronounce antioxidant activity with $\mathrm{IC}_{50}$ values of 58.71 and $8.83 \mathrm{ppm}$, respectively (Table 2), in comparison to vitamin C. Meanwhile, $n$-hexane (KSH) displayed no activity as an antioxidant. These results confirmed the corresponding active crude extract and fractions from $D$. suffruticosa wood bark with active fractions from other parts of $D$. suffruticosa, which have been reported previously.

Table 2. Antioxidant, total phenolic content (TPC), and total flavonoid content (TFC) data of D.suffruticosa extract and fractions

\begin{tabular}{|c|c|c|c|}
\hline & $\begin{array}{c}\text { TPC } \\
\text { (mg GAE/g extract) }\end{array}$ & $\begin{array}{c}\text { TFC } \\
\text { (mg RE/g extract) }\end{array}$ & $\begin{array}{c}\text { antioxidant activity } \\
\left(\mathrm{IC}_{50}, \mathrm{ppm}\right)\end{array}$ \\
\hline $\mathbf{M}$ & $236.17 \pm 14.61^{\mathrm{c}}$ & $12.06 \pm 0.39^{\mathrm{b}}$ & $<15.63$ \\
\hline KSH & $1.88 \pm 0.00^{\mathrm{a}}$ & $11.65 \pm 0.17^{\mathrm{a}}$ & $>1000$ \\
\hline KSC & $164.72 \pm 1.47^{\mathrm{b}}$ & $15.33 \pm 0.26^{\mathrm{c}}$ & 58.71 \\
\hline KSM & $254.34 \pm 16.86^{\mathrm{c}}$ & $12.34 \pm 0.29^{b}$ & 8.83 \\
\hline Vitamin C & - & - & 4.45 \\
\hline
\end{tabular}

Note: $\mathrm{M}=$ crude extract, $\mathrm{KSH}=n$-hexane fraction, $\mathrm{KSC}=$ chloroform fraction, $\mathrm{KSM}=$ methanol fraction; values are presented as mean $\pm \mathrm{SD}$; different letters within the same column indicate significant difference at $p<$ 0.05 
Previously, fractions reported from $D$. suffruticosa, which are considered as pronounce antioxidant, displayed other bioactivities, such as antibacterial (Kristiningrum et al., 2020), antiinflammatory (Abubakar et al., 2019), and cytotoxic against several cancer cell lines (Armania et al., 2013). Many studies have reported how antioxidant agent plays roles in other bioactivity mechanisms. Based on this, D. suffruticosa wood bark would also demonstrate interesting bioactivity.

The determination of total phenolic content (TPC) of crude extract and all fractions revealed that methanol fraction displayed the highest total phenolic content with $254.34 \pm 16.86 \mathrm{mg} \mathrm{GAE} / \mathrm{g}$ extract, followed by crude extract, chloroform, and $n$-hexane fractions (Table 2). In addition to that, TPC and TFC values between each fraction had significant differences statistically $(p<0.05)$. These results supported phytochemical data regarding the existence of phenolics in all fractions. Therefore, it may indicate that phenolics contained in the wood bark mostly were polar compounds. Despite that the TPC value of each fraction was relatively high, which was ranging from $1.88 \pm 0.00$ to $254.34 \pm 16.86$ $\mathrm{mg} \mathrm{GAE} / \mathrm{g}$ extract, the total flavonoid content (TFC) was lower; ranging from $11.65 \pm 0.17$ to $15.33 \pm$ $0.26 \mathrm{mg} \mathrm{RE} / \mathrm{g}$ extract. These data suggested that flavonoids were not a major group in phenolics. Further, the highest total flavonoid content (TFC) was shown in the chloroform fraction, indicating that most flavonoids in D. suffruticosa wood bark were semipolar compounds.

An observation on the chromatogram profile of antioxidant-active methanol and chloroform fractions to analyze the relationship between antioxidant and phenolic content was performed using a simple TLC-bioautography for antioxidant and DPPH reagent by comparing the chromatogram profile of each fraction on TLC plate. The phenolic spots were shown by the change of the spots' color to dark brownish yellow color on the TLC plate after being sprayed with $\mathrm{CeSO}_{4}(1.5 \% \mathrm{~b} / \mathrm{v})$ followed by heating on the hot plate (Marliyana et al., 2019). The phenolics are usually semipolar to polar compounds. In this case, the best eluent systems chosen were $n$-hexane-acetone (1:1) to give semipolar property for chloroform fraction (KSC) and chloroform-methanol (10:0.2) to polar fraction, methanol (KSM). The experiment result showed that most spots that appeared in these fractions gave bright spots after being sprayed with the DPPH reagent (Figure 3). It indicated that most compounds contained in both fractions possessed antioxidant activity (Cieśla et al., 2015). Further, based on the TLC-bioautography experiment, the spots, which gave positive results for phenolics, displayed bright color in DPPH assay. It indicated that those phenolics possessed antioxidant capacity. Compared to the $\mathrm{IC}_{50}$ and the chromatogram profile of the chloroform fraction, the methanol fraction was more active and possessed more antioxidant-phenolic spots. Therefore, it can be assumed that the strong antioxidant activity showed by the methanol fraction was caused by the existence of those phenolics.
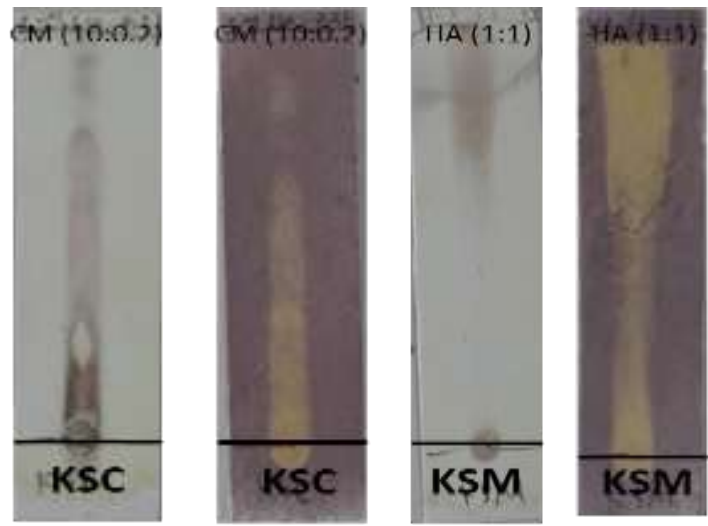

Figure 3.TLC bioautography and DPPH assay: chloroform fraction (KSC) eluted with chloroform-methanol (10:0.2) and methanol fraction (KSM) eluted with $n$-hexaneacetone (1:1) 
The relationship of phenolic content (TPC) or flavonoid content (TFC) with antioxidant activity was analyzed further using Pearson's product-moment correlation. The result revealed that there was a strong negative correlation between $\mathrm{TPC}$ and $\mathrm{IC}_{50}$ values with an $r$-value of -0.96 ( $p$-value $<0.05$ ) (Table 3), indicating that $\mathrm{IC}_{50}$ value of each fraction was inversely proportional to its TPC value. Since the antioxidant activity was stated as $\mathrm{IC}_{50}$ value, the lower $\mathrm{IC}_{50}$ value means a higher antioxidant activity. Thus, the result suggested that the higher TPC indicates a higher antioxidant activity. This finding was in agreement with the previous experiment using TLC bioautography. It was also relevant to previous research that mentioned the relationship between antioxidant and phenolic content (Kainama et al., 2020), which supports that antioxidant property shown by crude extract and all fractions was due to their phenolic content. Additionally, correlation between TFC and antioxidant in this study was moderate with $r=-0.43$ ( $p$-value $>0.05$ ) (Table 3 ). Thus, it gave another evidence that the exhibited antioxidant activity were likely caused by the phenolics rather than flavonoids.

Table 3. Pearson's correlation coefficient $(r)$ and linear relationship between antioxidant activity (AA), total phenolic content (TPC), and total flavonoid content (TFC) data of D.suffruticosa wood bark extract and fractions

\begin{tabular}{ccccc}
\hline & $\boldsymbol{r}$ & $\mathbf{R}^{2}$ & equation & $\boldsymbol{p}$ \\
\hline DPPH-TPC & -0.96 & 0.916 & $\mathrm{y}=-4.0476 \mathrm{x}+935.56$ & $<0.05$ \\
DPPH-TFC & -0.43 & 0.183 & $\mathrm{y}=-125.71 \mathrm{x}+1885.4$ & $>0.05$
\end{tabular}

Note: DPPH free radical scavenging was expressed as $\mathrm{IC}_{50}$ value (in $\mathrm{ppm}$ ). The lower $\mathrm{IC}_{50}$ value means a higher antioxidant activity (AA)

Many studies showed that most phenolics and flavonoids were known to possess antioxidant activity. Antioxidant-active phenolics were reported to be sensitive with DPPH assay (Bendary et al., 2013). This is because of the phenolics' ability to scavenge DPPH radical by its reactive oxygen and its ability to lose the $\mathrm{H}$ atom. All polyphenolics that were isolated previously from $D$. Suffruticosa (protocatechuic acid, gallic acid, vitexin, tiliroside, kaempferide, and kaempferol) were reported to exhibit antioxidant activity. Since phenolics dominated $D$. suffruticosa wood bark, it can be predicted that the antioxidant-active crude extract of $D$. suffruticosa wood bark and methanol fraction contain similar antioxidant-active phenolics previously reported.

\section{CONCLUSION}

This study successfully revealed the existence of alkaloids, phenolics, flavonoids, terpenoids, steroids, and saponins in the methanolic crude extract of $D$. suffruticosa wood bark. The pronounce antioxidant activity showed by the crude extract was also demonstrated by phenolic rich containing fraction, i.e., chloroform and methanol fractions. A strong correlation between antioxidant and phenolic content from all fractions suggested that phenolics were responsible for their antioxidant activities. Moreover, the isolation and structure elucidation of the active principles on methanol fraction should be conducted in future research plans to reveal the structure of the antioxidant-active phenolics. Hence, D. suffruticosa wood bark has the potential for a natural antioxidant source.

\section{ACKNOWLEDGEMENT}

The authors sincerely thank the Faculty of Teacher Training and Education (FKIP), Universitas Tanjungpura (UNTAN), for giving the PNBP FKIP DIPA research grant award for financial support. The authors also wish to thank Mr. Rudi Heryanto, Laboratorium Pusat Studi Biofarmaka, IPB University, for antioxidant assays. 


\section{REFERENCES}

Abubakar, S., Al-Mansoub, M. A., Murugaiyah, V., \& Chan, K. L. (2019). The phytochemical and anti-inflammatory studies of Dillenia suffruticosa leaves. Phytotherapy Research, 33(3), 660 675. https://doi.org/10.1002/ptr.6255

Ahmad, F. B., \& Holdsworth, D. K. (1995). Traditional medicinal plants of sabah, Malaysia part III. The rungus people of kudat. Pharmaceutical Biology, 33(3), 262-264. https://doi.org/10.3109/13880209509065377

Armania, N., Saiful, L., Noorhidayah, S., Safinar, I., Biau, J., Wei, K., Noreen, H., \& Hamid, A. (2013). Dillenia suffruticosa exhibited antioxidant and cytotoxic activity through induction of apoptosis and G 2 / M cell cycle arrest. Journal of Ethnopharmacology, 146(2), 525-535. https://doi.org/10.1016/j.jep.2013.01.017

Bendary, E., Francis, R. R., Ali, H. M. G., Sarwat, M. I., \& El Hady, S. (2013). Antioxidant and structure-activity relationships (SARs) of some phenolic and anilines compounds. Annals of Agricultural Sciences, 58(2), 173-181. https://doi.org/10.1016/j.aoas.2013.07.002

Chen, G. L., Fan, M. X., Wu, J. L., Li, N., \& Guo, M. Q. (2019). Antioxidant and anti-inflammatory properties of flavonoids from lotus plumule. Food Chemistry, 277, 706-712. https://doi.org/10.1016/j.foodchem.2018.11.040

Cië̈la, Ł. M., Waksmundzka-Hajnos, M., Wojtunik, K. A., \& Hajnos, M. (2015). Thin-layer chromatography coupled with biological detection to screen natural mixtures for potential drug leads. Phytochemistry Letters, 11, 445-454. https://doi.org/10.1016/j.phytol.2015.02.005

Dawood Shah, M., Seelan Sathiya Seelan, J., \& Iqbal, M. (2020). Phytochemical investigation and antioxidant activities of methanol extract, methanol fractions and essential oil of Dillenia suffruticosa leaves. Arabian Journal of Chemistry, 13(9), 7170-7182. https://doi.org/10.1016/j.arabjc.2020.07.022

Gacche, R. N., \& Jadhav, S. G. (2012). Antioxidant activities and cytotoxicity of selected coumarin derivatives: preliminary results of a structure-activity relationship study using computational tools. Journal of Experimental and Clinical Medicine, 4(3), 165-169. https://doi.org/10.1016/j.jecm.2012.04.007

Grigalius, I., \& Petrikaite, V. (2017). Relationship between antioxidant and anticancer activity of trihydroxyflavones. Molecules, 22(12). https://doi.org/10.3390/molecules22122169

Gunadi, D., Oramahi, H. ., \& Tavita, G. E. (2017). Studi tumbuhan obat pada etnis dayak di desa gerantung Kecamatan Monterado Kabupaten Bengkayang. Jurnal Hutan Lestari, 5(2), 425-436. http://eprints.umm.ac.id/44014/\%0Ahttp://jurnal.untan.ac.id/index.php/jmfkh/article/view/20089/ 16490

Kainama, H., Fatmawati, S., Santoso, M., Papilaya, P. M., \& Ersam, T. (2020). The relationship of free radical scavenging and total phenolic and flavonoid contents of Garcinia lasoar PAM. Pharmaceutical Chemistry Journal, 53(12), 1151-1157. https://doi.org/10.1007/s11094-02002139-5

Kessler, A., \& Kalske, A. (2018). Plant secondary metabolite diversity and species interactions. Annual Review of Ecology, Evolution, and Systematics, 49, 115-138. https://doi.org/10.1146/annurev-ecolsys-110617-062406

Kristiningrum, N., Amaliyah, E. A., \& Pratoko, D. K. (2020). Phytochemical screening, antioxidant and antibacterial activities of ethanol extract and fractions of Aleurites moluccana (L.) willd. leaves. Tropical Journal of Natural Product Research, 4(11), 895-898. https://doi.org/10.26538/tjnpr/v4i11.9

Marliyana, S. D., Mujahidin, D., \& Syah, Y. M. (2019). Transformation reaction of prenylated chalcone of pinostrobin derivative and their antibacterial activity. IOP Conference Series: Materials Science and Engineering, 509(1), 22-27. https://doi.org/10.1088/1757$\underline{899 X / 509 / 1 / 012133}$ 
Salazar-aranda, R., P, L. A., Joel, L., \& Alan, B. A. (2011). Antimicrobial and antioxidant activities of plants from Northeast of Mexico. Evidence-Based Complementary and Alternative Medicine, 2011, 536139. https://doi.org/10.1093/ecam/nep127

Sticher, O. (2008). Natural product isolation $\dagger$. Natural Product Reports, 25(3), 517-554. https://doi.org/10.1039/b700306b

Tor, Y. S., Yazan, L. S., Foo, J. B., Armania, N., Cheah, Y. K., Abdullah, R., Imam, M. U., Ismail, N., \& Ismail, M. (2014). Induction of apoptosis through oxidative stress-related pathways in MCF-7 , human breast cancer cells, by ethyl acetate extract of Dillenia suffruticosa. BMC Complementary and Alternative Medicine, 14, 55. https://doi.org/https://doi.org/10.1186/1472$\underline{6882-14-55}$

Wiart, C., Mogana, S., Khalifah, S., Mahan, M., Ismail, S., Buckle, M., Narayana, A. K., \& Sulaiman, M. (2004). Antimicrobial screening of plants used for traditional medicine in the state of Perak, Peninsular Malaysia. Fitoterapia, 75, 68-73. https://doi.org/10.1016/j.fitote.2003.07.013

Yazan, L. S., \& Armania, N. (2014). Dillenia species: A review of the traditional uses , active constituents and pharmacological properties from pre-clinical studies. Pharmaceutical Biology, 52(7), 890-897. https://doi.org/10.3109/13880209.2013.872672 